Research Article

\title{
Research on Virtual Display of Wetsuit Based on CLO3D
}

\author{
Haifeng Wang and Joung Hyung Cho $\mathbb{D}$ \\ Department of Marine Design Convergence Engineering, Pukyong National University, Busan 612022, Republic of Korea
}

Correspondence should be addressed to Joung Hyung Cho; 202056741@pukyong.ac.kr

Received 7 September 2021; Accepted 19 October 2021; Published 15 December 2021

Academic Editor: Rahman Ali

Copyright (c) 2021 Haifeng Wang and Joung Hyung Cho. This is an open access article distributed under the Creative Commons Attribution License, which permits unrestricted use, distribution, and reproduction in any medium, provided the original work is properly cited.

\begin{abstract}
In order to overcome the problems of low signal-to-noise ratio in the information output interface and long time for information synthesis in the traditional virtual display method of clothing, a CLO3D-based virtual display method for wetsuit is designed in this study. The proposed method works as follows. Firstly, it analyzes the categories and functional characteristics of the wetsuit and the virtual display process of the CLO3D software. In the second step, the design of the proposed method for the process of data collection and fusion of the wetsuit design is made. In the subsequent steps, human model is established, designs are made for the style and modeling, simulation is made for the pattern and color of the wetsuit fabric, and dynamic display is made. Experimental results show that the signal-to-noise ratio (SNR) of the information output interface of the proposed method is above $75 \mathrm{~dB}$, and the maximum SNR can reach $80.5 \mathrm{~dB}$, and the information synthesis time varies between $32 \mathrm{~min}$ and $47 \mathrm{~min}$, indicating that the proposed method is more efficient and effective.
\end{abstract}

\section{Introduction}

With the development of society and the continuous improvement of living standards, people are increasingly pursuing entertainment and leisure sports in addition to materials. With the continuous and diversified development of China's leisure and entertainment market, diving is more and more favored by consumers [1]. People's requirements for diving equipment and facilities have gradually increased, and the research on diving equipment has gradually increased. Diving equipment is used to prevent hypothermia due to the rapid loss of body temperature while diving and also to protect the diver from rocks or harmful animals and plants. Diving suit is a kind of diving equipment; it is an important equipment of diving operation, widely used in military and civilian diving.

The application of virtual reality in the garment industry began in the 1980s. It is a three-dimensional garment virtual display technology based on virtual reality and digital simulation technology, taking garment sample design and sample production process as inspiration [2]. Nowadays virtual technology has covered all fields of today's society, and at the same time, it provides a new development opportunity for the creative expression of fashion designers. The application of virtual design technology has changed the limitations of paper, brush and pigment required by traditional design and painting, and will not be repainted for the adjustment of clothing style and color required by customers. Virtual clothing design is to carry out conception, design, test, and analysis of clothing in the computer so as to improve the decision-making level of clothing in time, quality, cost, and service and achieve the purpose of global optimization and one-time development.

To this end, a virtual clothing display method based on holographic projection technology is designed in [3]. Aiming at the problem of poor spatial sense in virtual clothing display, this method analyzes the principle of holographic projection technology and its application in fashion design, fashion dynamic show, and other aspects. In addition, a garment display method combining 360-degree holographic projection technology with virtual garment display was proposed. A simple 360-degree holographic projection device was made by this method, and one dynamic virtual garment and two static virtual garments were displayed. This method can be applied to virtual costume exhibition such as costume museum and costume 
conference. Reference [4] designs a physics-based interactive virtual costume animation simulation display method, which designs effective storage methods according to the content characteristics of models and animation resources and provides an interactive control parameter adjustment interface. Then, the implicit constraint dynamics system, collision detection acceleration, and constraint optimization calculation model were constructed, and the cloth animation calculation was carried out. Finally, Unity $3 \mathrm{D}$ engine is used to render the simulation data to show the simulation effect under different scenes.

However, in the practical application process, it is found that the above traditional daily display screen has the disadvantages of low signal-to-noise ratio and long timeconsuming virtual display process. To solve these problems, this study designed a new virtual display method of wetsuit based on CLO3D software.

Motivation and aim of the study are to solve the problems of low signal-to-noise ratio in the information output interface and long time for information synthesis in the traditional virtual display method of clothing. The specific objective is to design a virtual display method of wetsuit on the basis of CLO3D.

The contributions of this study are as follows:

(i) Efficient analysis of the categories and functional characteristics of the wetsuit and the virtual display process of the CLO3D software

(ii) Design of the proposed method for the process of data collection and fusion of the wetsuit design

(iii) Establishment of the human model and simulation of the pattern and color of the wetsuit fabric

\section{Correlation Analysis of Wetsuit}

Diving suit is a kind of diving equipment, which is used to prevent hypothermia caused by the rapid loss of body temperature during diving and also to protect divers from damage from rocks or harmful animals and plants [5]. Diving suits were invented long before space suits. The ocean, this mysterious, blue field, has been deeply attracted to human beings, inspiring our desire to explore. In order to prevent possible dangers, inventors invented various protective wetsuits for divers, greatly improving the safety of underwater work.

2.1. The Development of Wetsuits. The first true wetsuit was made by Italian physicist Alfonso Perelli in the early sixteenth century. His wetsuit was made of waterproof leather and metal helmet and used a snorkel to breathe. In the seventeenth and eighteenth centuries, the British designed a number of wetsuits, which allowed divers to stay underwater for a long time, and were the closest to modern wetsuits [6].

In the 19th century, the famous engineer Augustus Siebe switched to a new tarpaulin material for his wetsuits. For the next hundred years, wetsuits were mainly used for engineering, so their design did not change much. Later, British inventor John Lethbridge began experimenting with making wetsuits out of rubber and designed a circulating breathing device with compressed air, which made the diver less constrained by the length of the snorkel tube and less troublesome to supply with oxygen.

In the 20th century, in response to the demands of war, the French inventor Yves Leprel designed another device for making oxygen with compressed air, which was pumped purely through a hose into the diver's mouth and then out through a one-way valve through a short tube on the other side. The British and Italian navies developed new circulatory breathing equipment and trained frogmen during World War II. International designers then began trying to use the amount of mixed gas to increase the amount of time divers spent underwater, an effort that continues to this day.

With the development of material science and technology, the development of foam neoprene, this material is a very perfect wetsuit material, it has a certain flexibility, in the temperature of the sea can also play a warm role, and it also has a certain buoyancy, less resistance. With the global popularity of diving, neoprene wetsuit is divided into semidry wetsuit and wet wetsuit, while the tailoring of wetsuit is constantly keeping up with the development of The Times, and even becomes the fashionable clothing for diving participants [7].

2.2. Categories of Wetsuits. The diving suits are dry, wet, and semidry. When wearing a dry wetsuit, the body is completely isolated from the water, depending on the temperature of the water, can be inside the sweater to strengthen heat preservation. You have to be trained to use it for diving in cold climates. Dry wetsuits are much higher than wet wetsuits because they require special waterproof zippers and other accessories, such as dry diving inflatable exhaust devices. The use of a dry suit requires special training to learn how to control and use the air. Dry diving suit maintenance and maintenance method is to dive dry diving suit to soak water and avoid sunlight exposure, stored in a ventilated and cool place as far as possible. Zippers should be lubricated frequently and cannot be folded for a long time to prevent irreparable wrinkling of foam synthetic rubber [8].

Wetsuit is the most commonly used diving suit, made out of foam rubber, with a general thickness from $1.5 \mathrm{~mm}$ to $10 \mathrm{~mm}$ above. Wearing a diving suit will heat up quickly and be isolated by active bubbles, which can prevent body heat from dissipating. Wearing a wet suit can make the exchange between inside and outside faster, and the isolation effect will be better. The wetsuit is made of foamed rubber or nylon cloth and worn next to the body. The wetsuit must be fitted to the skin, and the small amount of water that enters the suit will not flow between the skin and the suit to maintain body temperature.

Half-dry suit by the neck, wrist, and ankle rubber ring seal makes the body keep dry with warm; its basic characteristics are the same as those of dry diving suit; only the feet, hands, and head are directly exposed to water or wearing wet caps, gloves, and other measures to keep warm, and it is mainly used in diving travel, fishing, underwater construction, etc.

\subsection{The Functional Characteristics of the Wetsuit}

(a) Heat Preservation. All three types of wetsuits have the same functionality in some way. First, all three types of 
wetsuits must have some thermal insulation. Due to the cold underwater environment, the data show that the average temperature of the sea surface is between $-1.7^{\circ} \mathrm{C}$ and $27^{\circ} \mathrm{C}$. Studies have shown that the human body feels comfortable at room temperature of $25^{\circ} \mathrm{C}$, but it feels cool at water temperature of $25^{\circ} \mathrm{C}$. Even at $25^{\circ} \mathrm{C}$, subjects without wetsuits were rarely able to stay in water for more than an hour. For the sake of human health, water temperature above $27^{\circ} \mathrm{C}$ does not require wearing diving suits; wear wet wetsuit below $20^{\circ} \mathrm{C}$; dry wetsuits are required if the water temperature is below $10^{\circ} \mathrm{C}$. Therefore, the wetsuit must have certain thermal insulation performance [9].

(b) Fitness. Dry wetsuit fit requirements are not as strict as wet wetsuit; wet wetsuit requires clothing and human body as much as possible to fit, which is determined by its thermal principle. The principle of wet wetsuit is as follows: when the water layer between the human body and the wetsuit does not flow, the water layer between the human body and the wet wetsuit can form a warm water bath environment around the human body so that the body surface heat loss is the least for the wet wetsuit to achieve the effect of warmth.

(c) Motility. In addition to the insulation and fit of the diving pants, the sports of the diving pants are also very important. Compared with dry-type wetsuits, wetsuits have no exhalation device. They are light, flexible, and comfortable to wear. However, if they are in a bubble wetsuit made of neoprene, they will compress the pores of the neoprene foam when diving to deep external water pressure. Its thickness becomes thinner.

\section{Analysis of Clothing Digitization Technology of CLO3D Platform}

CLO3D virtual clothing technology is the combination of graphics and simulation technology and then through the simulation of clothing to complete the structure of clothing performance so that we can intuitively see the structure and form of clothing.

With the development of technology in the information age, CLO3D platform virtual display technology of clothing can realize more complete pattern making, process structure, and effect drawing of clothing. CLO3D software has been significantly improved in quality and efficiency, and the perfect duplication of clothing structure in technology has been realized. The simulation effect of simulated clothing needs to be displayed from multiple angles [10].

3.1. Introduction to CLO3D Software. CLO3D software is from the South Korean CLO company CLO3D software; the software is a strong simulation clothing virtual software. The software also includes boilerplate window, virtual avatar window, object window, and property window of boilerplate drawing, as shown in Table 1.

In the CLO3D software, the effect of multiperspective presentation method is used to realize the omnidirectional and multiangle observation of virtual clothing design works so as to find out the potential problems that may exist in the design works. This all-round three-dimensional effect brings modern visual appreciation to clothes and gives us more visual perspectives. It can accurately express the style and shape of clothing structure by transforming two-dimensional plates into three-dimensional ones [11].

With the rapid development of information technology in recent years, virtual reality technology can also be applied in the current fashion design industry. The University of Tubingen used this technology to simulate the hanging effect of silk and cotton fabric, the University of North Carolina also used this technology to control the composite bending of the fabric in virtual clothing, and various universities studied to establish the human body model system to realize the efficient transformation of two-dimensional and three-dimensional. The $3 \mathrm{D}$ virtual avatar fitting and 2D sample drawing can be designed and modified at the same time, and the data can be synchronized. And in the virtual avatar window or template window to select objects (virtual avatar, clothing, and template), you can select multiple templates or internal graphics in the object window, and then through the property window, you can adjust the attribute value.

CLO3D is $3 \mathrm{D}$ clothing virtual software. It can integrate the building of human model, 2D sample, 3D garments virtual stitching, fabric simulation, and dynamic virtual display. Designs that are usually difficult for designers to express can be presented through three-dimensional effects. For the board division, two-dimensional and three-dimensional transformation model can be adjusted and modified to achieve the effect of dress fit. Virtual sample clothing production can be realized for garment enterprises, which greatly saves the production cost of garment enterprises. At the same time, virtual fitting and other functions can be used to create clothes for consumers based on their own body type [12].

3.2. Virtual Presentation Process of CLO3D Software. In this paper, CLO3D was used as a virtual display platform for wetsuit. The software's interface includes both a virtualized window and a boilerplate window, showing a 3D model and a $2 \mathrm{D}$ boilerplate of the wetsuit, respectively. In the process of design modification, the data between the two windows can be synchronized; the operation is fast and intuitive. After the virtual design effect is determined, the $2 \mathrm{D}$ sample data information contained in it can be shared with the pattern maker to make the plate, and even directly used in the production of sample clothes, which greatly saves the development cost in the early stage of the design and installation of wetsuit. Generally speaking, the process of clothing design based on CLO3D is as follows:

(i) Step 1. The first step is to build a parameterized human model; that is, the human model can change with the human size data. The data of the initial mannequin can be obtained by measuring the body.

(ii) Step 2. The second step is to draw the two-dimensional garment basic template and sew it 
TABLE 1: CLO3D windows and functions.

\begin{tabular}{lr}
\hline Window & Function \\
\hline Sample window & Drawing of sample, setting cutting line, editing cloth image \\
Avatar window & You can wear virtual clothing; you can move to create animation \\
Physical window & Virtual object images and templates to create and edit fabric points \\
The properties window & Display sample, clothing, material, physical properties, and other basic information and settings \\
\hline
\end{tabular}

virtually onto the human body model to generate the three-dimensional garment model that meets the human body. Then, through repeated interactive modifications, the three-dimensional garment design effect is effectively checked.

(iii) Step 3. The third step is to match the color of the fabric, adjust the properties of the fabric, and test the comfort of the garment.

(iv) Step 4. The fourth step is the virtual wetsuit static and dynamic display.

\section{Virtual Display Design of Diving Suit Based on CLO3D}

The subsequent sections further describe the details of data collection and fusion, building a mannequin, designing style model, simulating wetsuit fabric pattern and color matching, and dynamic virtual display.

\subsection{Data Collection and Fusion Process Related to Wetsuit.} Before constructing the virtual display method of the wetsuit, firstly, it is necessary to design the sensor unit, use multiple sensors to collect the relevant data of the wetsuit, implement the corresponding data fusion processing, and then send the fusion processing results to the background of CLO3D software for specific processing.

In general, when various sensors collect temperature, pressure, color, shape, and other data, due to the interference of the target environment and the sensor itself, the data collected by various sensors are prone to many problems, such as error data, redundant data, packet loss data, etc. [13]. In order to avoid the above problems, it is necessary to carry out the fusion processing of all kinds of sensor data collected. The fusion process is shown in Figure 1.

In Figure 1, sensors $a, b$, and $c$ are different types of sensors, respectively, and the data collected by each sensor is represented by $z_{a}, z_{b}$, and $z_{c}$ in sequence. The weight coefficient interval of data fusion of each sensor is $[0,1]$, and the series $\omega_{i j}$ represents the data array, where $i$ represents the series of data fusion, where the value of $i$ can be 1 or 2 . The data fusion process is divided into two levels. The first level is the fusion of the data collected by the same type of sensors in a specific period of time, and the second level is the fusion of the data collected by all types of sensors.

Taking sensor $a$ as an example, the sensor collects data $z_{a n}$ at a time interval of $0.2 \mathrm{~min}$ and fuses and processes the data collected by the sensor once every hour. The first-level fusion processing value of the obtained data is represented by $e\left(z_{a}\right)$. Then, the first-level fusion processing values of the data collected by the other two sensors are represented by

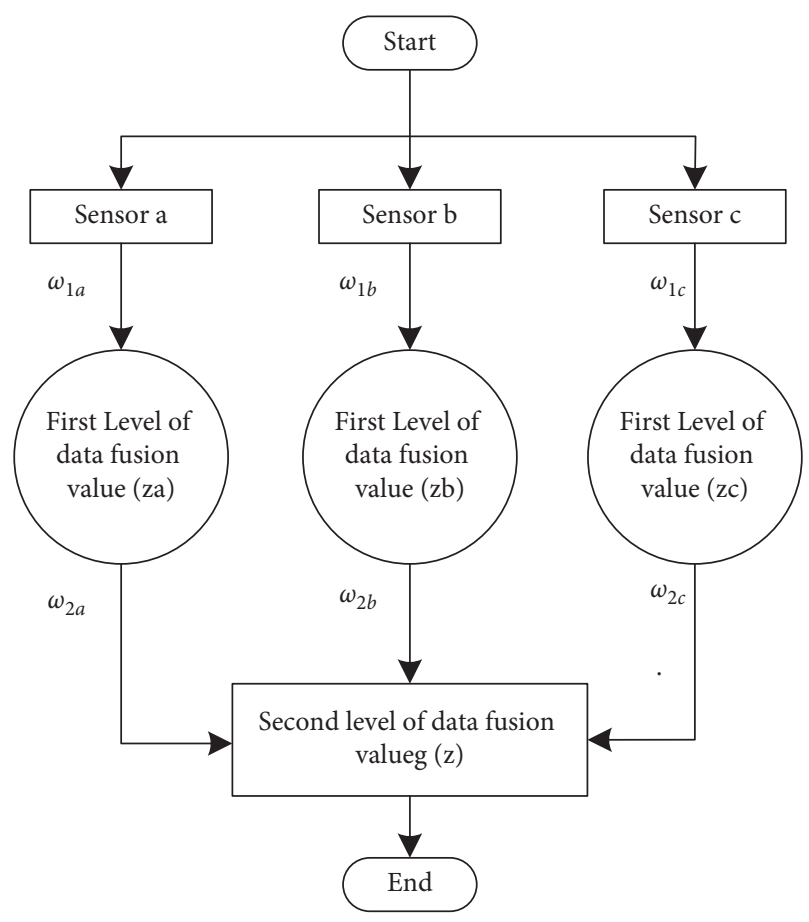

Figure 1: Multisensor data fusion processing process diagram.

$e\left(z_{b}\right)$ and $e\left(z_{c}\right)$, respectively. The second-level fusion is carried out on the first-level fusion processing values of the three sensors, and the final fusion processing result $g(z)$ is obtained. The two-level fusion processing can be expressed as

$$
\left\{\begin{array}{l}
e\left(z_{j}\right)=\sum_{j=a}^{12} \omega_{1 j} z_{i j}, \quad(j=a, b, c) \\
g(z)=\sum_{i=1}^{3} \omega_{2 j} e\left(z_{j}\right)
\end{array}\right.
$$

4.2. Building a Mannequin. A mannequin is the basis of clothing design, but also the basis of clothing virtual display, its size, and posture are particularly important in clothing virtual design, should have static and dynamic clothing display function. Static display of clothing is equivalent to the human body standing still, which is convenient to observe the drape effect of clothing, while dynamic display is used to observe the overall effect of clothing during the movement of models. The dynamic display function can be based on the parameterized human model provided by CLO3D, and it adds the action command to make it move so as to simulate the scene when the human movement. In 
addition, in order to enrich the image and movement of the model, you can use 3D software such as MAYA or 3DS MAX to create a human model. Mannequins are divided into three levels: skeleton, muscle, and skin. By defining the movement of the skeleton, muscles and skin are driven to get the moving mannequins [14].

In this paper, based on the human model provided by CLO3D, according to the design needs and the physical features of customers, select a suitable model base in the model library, and set its height, shoulder width, chest circumference, waist circumference, arm circumference, and other dimensions; that is, the modeling work of human model has been completed. Setting a 3D model is shown in Figure 2.

4.3. Design Style Modeling. The dynamic effect of the model's dress is of great reference value to the design of the diving suit. For the design of the wetsuit based on the 3D model, it is necessary to draw the two-dimensional sample of the clothing first, and then sew the two-dimensional sample onto the model to form a $3 \mathrm{D}$ wetsuit, which is equivalent to wearing the clothing on the human body in real life.

The implementation steps based on CLO3D are as follows:

(a) Step 1. Draw the template. In the sample window, draw the two-dimensional diving suit sample according to the human body model, and the data information is displayed on the sample accurately.

(b) Step 2. Place the sample. After the sample is drawn, execute the "synchronize" command, and the sample will appear in the virtual model window. Then, select the "arrangement point" mode to arrange the surrounding sample so that the sample is closer to the model.

(c) Step 3. Suture. Select the smart sewing tool to sew the corresponding part virtual, and finally execute the "simulation" command; the garment piece is worn on the 3D model. Suture types are shown in Figure 3.

(d) Step 4. Modify the version. After review, adjustments can be made in the two-dimensional boilerplate window, and synchronous modifications can be made in the virtual avatar window.

4.4. Simulate Wetsuit Fabric Pattern and Color Matching. Modern clothing design requires personalized expression effect; fabric pattern design is one of the key factors. While in the design of clothing fabric pattern, designers often draw lessons from a large number of traditional art graphics or modern images, symbols, patterns, etc., and then simplify and summarize them in practical use. Obviously, the use of computer technology can greatly improve work efficiency [15]. In this paper, the image processing software Photoshop was first used to process the structure and color of the wetsuit, and then it was used as fabric pattern in the wetsuit design. The specific steps are as follows: (a) Fabric Pattern Design. The hue and saturation of the pattern were adjusted to coordinate with the background color in the way of "matting" with the relevant LOGO through Photoshop and save the processed fabric pattern in JPG format.

(b) Fabric Filling. The designed fabric pattern was filled into the sample of the wetsuit in CLO3D software, and the density of the fabric and the size of the pattern were edited.

(c) Fabric Material Simulation. The physical properties of the fabric, such as fabric color, reflectivity, transparency, saturation, softness, strength, etc., were adjusted so as to achieve a realistic simulation effect of the fabric. The texture parameters of the wetsuit fabric in the property window were set so that the wetsuit display effect has a stronger threedimensional sense.

(d) Fabric Color Matching. By changing the fabric pattern and color, the pattern and color matching in the virtual wetsuit design can be realized quickly, and a changeable effect can be obtained.

4.5. Dynamic Virtual Display. The dynamic virtual display of wetsuit is a form of expression to show the wearing effect of wetsuit with people as the carrier. It is a powerful means to show the design idea and design level of wetsuit designers. Its purpose is to better let customers see the style and characteristics of wetsuit itself and more truly reflect the function and aesthetic characteristics of wetsuit.

The dynamic virtual display of wetsuit plays an important role in the design of wetsuit. Even if the designed virtual wetsuit works well on the static human body, it cannot guarantee that the dynamic display will have the same good effect. Only through dynamic display can we fully observe the fit degree of the wetsuit, the drape and texture of the fabric, and the overall dressing effect.

The virtual dynamic display is not constrained by physical conditions and space environment, which not only eliminates the large amount of preparatory work required to plan a wetsuit display, but also saves the high cost of hiring professional models. In addition, in the virtual wetsuit display, the digital mannequin can show the design effect at any time so that bad schemes can be eliminated in the design stage, saving the cost of style development and shortening the development cycle. In addition, the virtual display can be published through the network to realize the close combination of design and sales.

In this paper, CLO3D software is used to realize the dynamic virtual display of the wetsuit. Firstly, the virtual model is loaded with actions in CLO3D, and the animation video is recorded. The project file is generated after editing, and then the dynamic display is performed. The specific steps are as follows:

(a) Step 1. Add the action file: click the file in the avatar window to open the action command and load the action library. At this time, the action key in the 


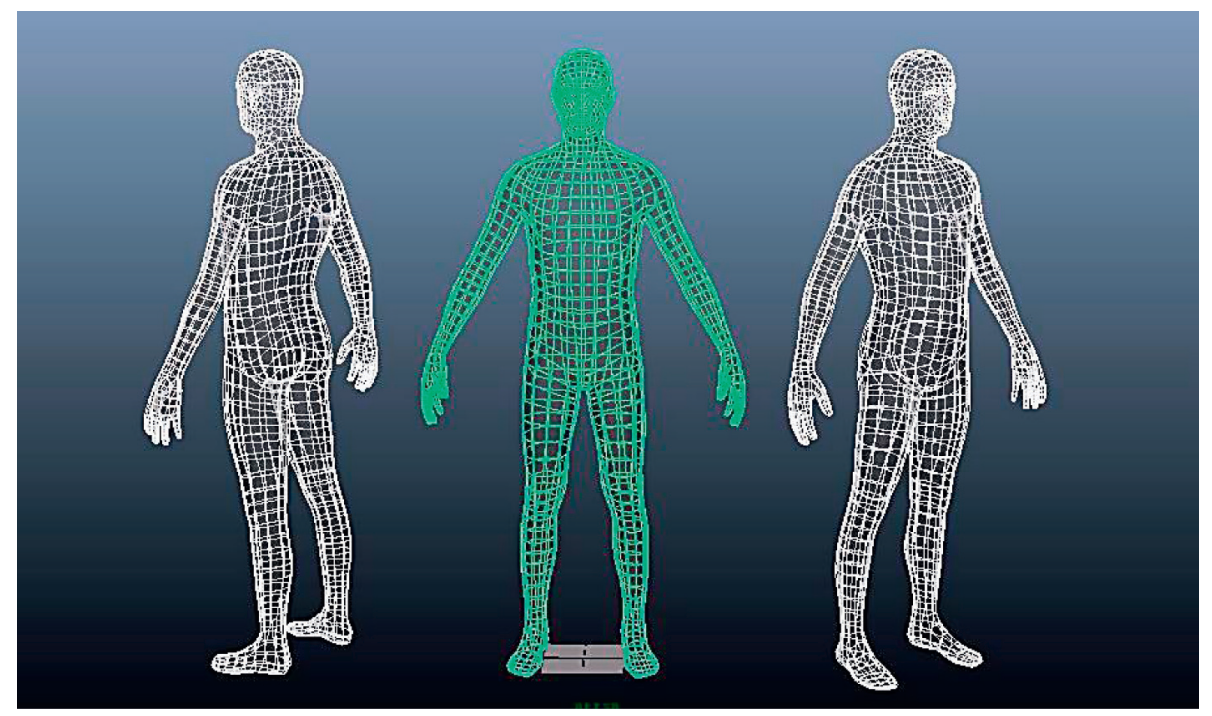

Figure 2: Setting a 3D model.

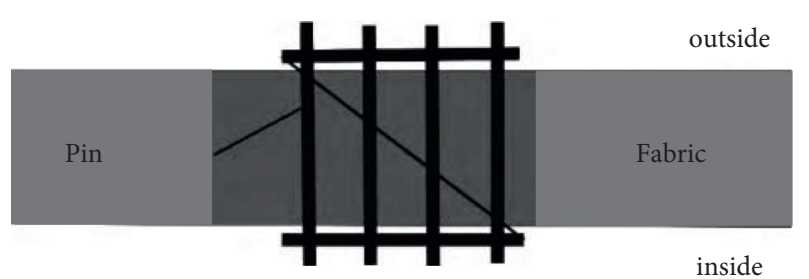

(a)

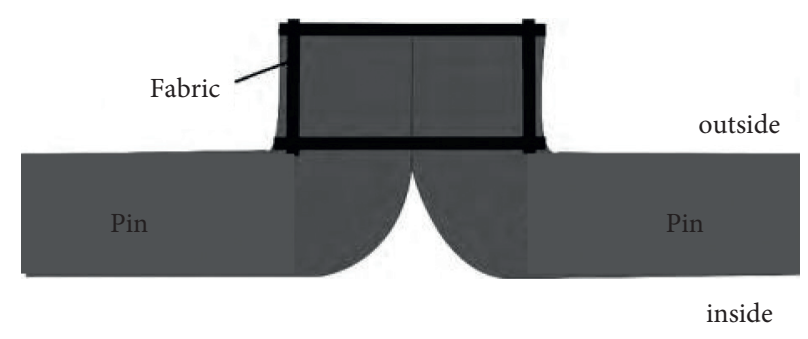

(b)

Figure 3: Schematic diagram of sewing method of diving suit. (a) Flat seam. (b) Wrapping seam.

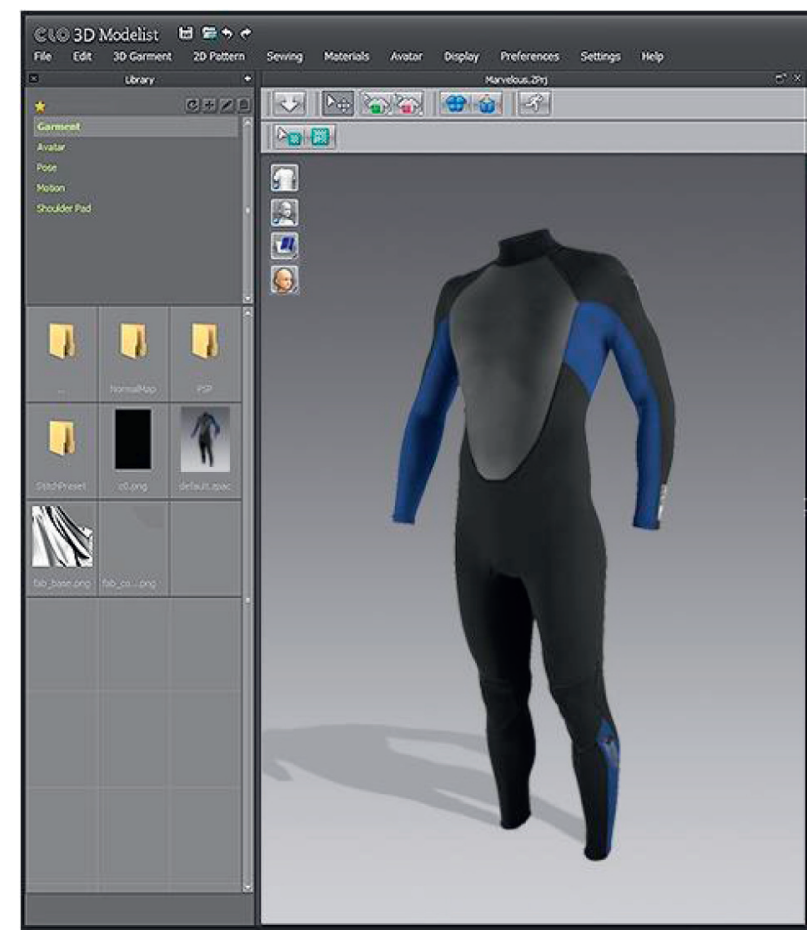

Figure 4: A mannequin in a wetsuit. 
toolbar is automatically highlighted, indicating that there is movement data in the scene. Click the simulation key in the toolbar, and you can see the picture of the mannequin wearing the diving suit. This step interface is shown in Figure 4.

(b) Step 2. Record the video file: click the record button to record the Animation of the moving virtual model until all the actions are played and save the Animation as an Animation file.

(c) Step 3. Save the project file: open the saved Animation file, adjust and set the playing part for editing, and then save it as the project file.

(d) Step 4. Render. Close the 3D virtual model, adjust the render wetsuit texture, and save it as an image file;

(e) Step 5. Dynamic virtual display. Click the virtual clothing in the interface Clothes to start the interactive THREE-DIMENSIONAL virtual display, and the environmental information can be interactive adjusted according to the requirements of the display effect of the wetsuit. The final virtual display interface is shown in Figure 5.

\section{Experiment and Research}

In order to verify the practical application performance of the above designed CLO3D-based virtual display method for wetsuit, the following experiments are designed for verification.

The simulation verification experiment is carried out in the MATLAB simulation platform, and the quality of the simulation experiment is improved by using the features of MATLAB GUI, such as simple, intuitive, easy to adjust, and easy to modify the program.

In order to enhance the credibility and effectiveness of the experimental results, the method of this article, the virtual clothing display method based on holographic projection technology, and the physical simulation display based on this method can use interactive virtual clothing animation as an experimental comparison. The noise ratio and synthesis are taken from two angles, and the performance verification is completed in combination with the method in this paper.

Firstly, the SNR of the information output interface is taken as the index to test the performance of different methods, and the results are shown in Figure 6.

Through the analysis of the results shown in Figure 6, it can be seen that the SNR of the information output interface of the method in this paper is above $75 \mathrm{~dB}$, and the maximum can reach $80.5 \mathrm{~dB}$, which is significantly higher than the two traditional methods. The SNR curves of the information output interface of the virtual clothing display method based on holographic projection technology and the physical interactive virtual clothing animation simulation display method are below the curves of the method in this paper, and the changes are obvious. It can be shown that the ratio of useful information to noise in the virtual display results of wetsuit obtained by the method in this paper is

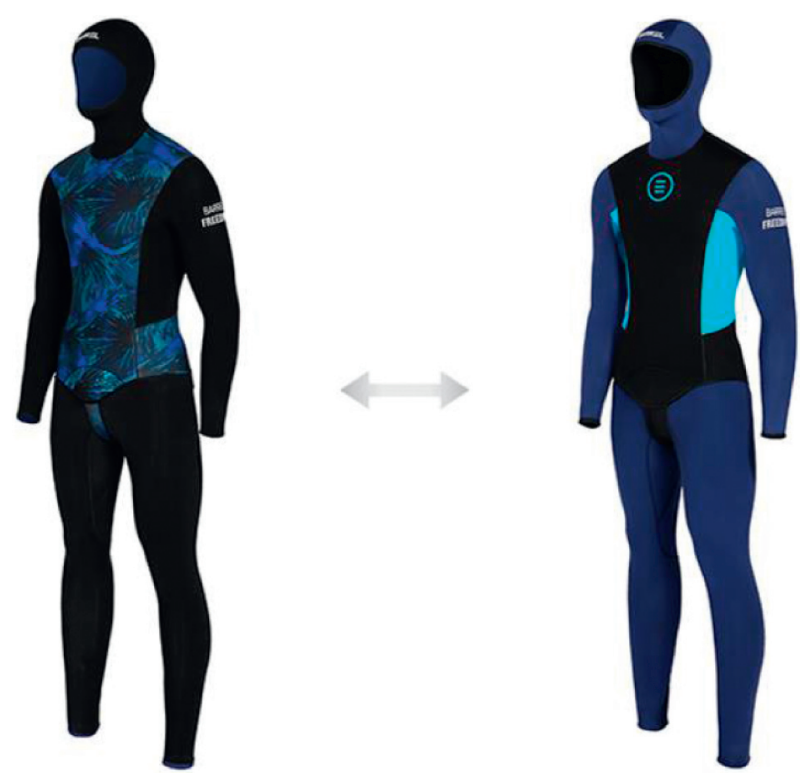

FIgURE 5: Wetsuit virtual display interface.

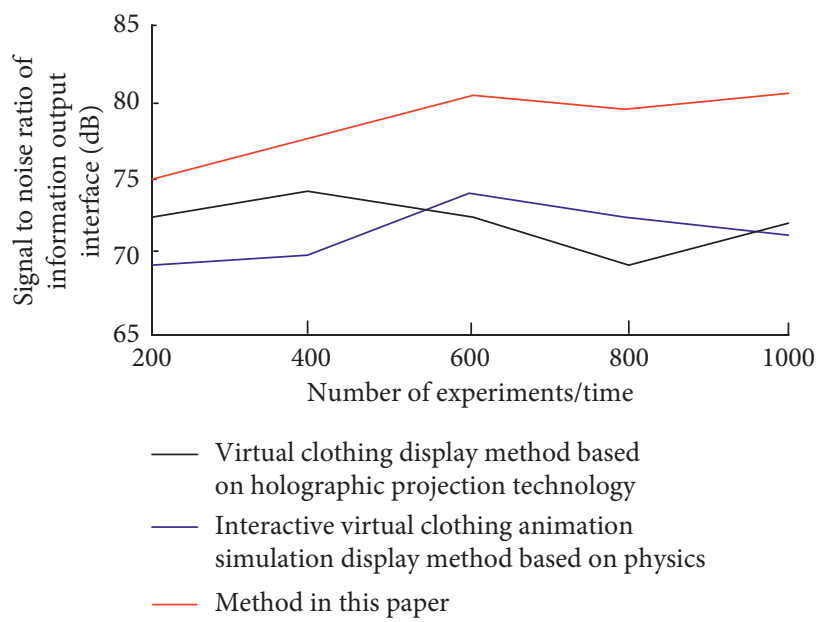

FIGURE 6: Different methods of information output interface SNR.

higher, which also indicates that the method in this paper is more effective.

Then, the information synthesis time of different methods was counted, and the results were shown in Table 2.

By comparing the results in Table 2, it can be seen that after the virtual garment display method based on holographic projection technology is applied, with the increasing number of tests, its information synthesis time fluctuates between $73 \mathrm{~min}$ and $96 \mathrm{~min}$. After the application of the physics-based interactive virtual clothing animation simulation display method, the information synthesis time fluctuates between $60 \mathrm{~min}$ and $94 \mathrm{~min}$ with the increase of the number of tests. After the application of the method in this paper, the information synthesis time fluctuates between $32 \mathrm{~min}$ and $47 \mathrm{~min}$ with the increasing number of tests. Through the above comparison, it can be seen that the information synthesis time of the method in this paper is less, indicating that the method has higher efficiency. 
TABLE 2: Statistical results of information synthesis time of different methods ( $\min )$.

\begin{tabular}{lccc}
\hline $\begin{array}{l}\text { Number of } \\
\text { experiments/ } \\
\text { times }\end{array}$ & $\begin{array}{c}\text { Virtual clothing } \\
\text { display method } \\
\text { based on } \\
\text { holographic } \\
\text { projection } \\
\text { technology }\end{array}$ & $\begin{array}{c}\text { Interactive } \\
\text { virtual clothing } \\
\text { animation } \\
\text { simulation } \\
\text { display method } \\
\text { based on physics }\end{array}$ & $\begin{array}{c}\text { Method } \\
\text { in this } \\
\text { paper }\end{array}$ \\
\hline 200 & 74 & 80 & 32 \\
400 & 96 & 71 & 36 \\
600 & 73 & 60 & 47 \\
800 & 73 & 94 & 44 \\
1000 & 88 & 82 & 45 \\
\hline
\end{tabular}

\section{Conclusion}

In order to solve the problems of low signal-to-noise ratio in the information output interface and long time for information synthesis in the traditional virtual display method of clothing, a virtual display method of wetsuit based on CLO3D was designed in this study. The specific display method was designed through the process of data collection and integration of the wetsuit, the establishment of the human model, the design of styles and modeling, the simulation of the wetsuit fabric pattern and color matching, and the dynamic display. The experimental results show that the signal-to-noise ratio of the information output interface of this method is above $75 \mathrm{~dB}$, and the maximum can reach $80.5 \mathrm{~dB}$, and the information synthesis time fluctuates between $32 \mathrm{~min}$ and $47 \mathrm{~min}$, indicating that the design expectations are achieved.

In the research area of wetsuit body dynamic measurements, there are still many gaps existing in the real and virtual experiments. Therefore, in the future manuscripts, we are planning to address these issues and provide the deformed digital twin for wetsuit pattern design. Therefore, the study is aimed to design and optimize the wetsuit and further improve the efficiency of manufacturers and their evaluation in our future publications. Furthermore, in the future, we plan for more extended comparison and evaluation against the latest research in the area.

\section{Data Availability}

The data used to support the findings of this study are available from the corresponding author upon request.

\section{Conflicts of Interest}

The authors declare that they have no conflicts of interest.

\section{Acknowledgments}

This work was supported by a grant from Brain Korea 21 Program for Leading Universities and Students (BK21 FOUR) MADEC Marine Designeering Education Research Group.

\section{References}

[1] Y. E. Hailian and Y. Chen, "Application OF three-dimensional virtual fashion design,” Design, vol. 15, no. 5, pp. 107-119, 2018.

[2] H. Lai and S. Lee, "The application of artificial intelligence and VR technology in clothing store display design," IEEE Access, vol. 13, no. 9, p. 1, 2020.

[3] H. Zhang and J. Chen, "Research of holographic projection technology on virtual clothing display," Melliand China, vol. 48, no. 11, pp. 55-59, 2020.

[4] M. Shi, J. Wang, T. Mao, and Y. Liu, "Physically-based interactive virtual clothing animation simulation," Journal of System Simulation, vol. 30, no. 7, pp. 2583-2592, 2018.

[5] G. Q. Chen and J. M. Sun, "Research progress of non-contact 3D body measurement technology and its application in garment industry," China Textile Leader, vol. 5, no. 12, pp. 81-84, 2018.

[6] B. K. Kim, G. Kim, and S. Y. Lee, "Style-controlled synthesis of clothing segments for fashion image manipulation," IEEE Transactions on Multimedia, vol. 22, no. 2, pp. 298-310, 2019.

[7] I. Santesteban, M. A. Otaduy, and C. Dan, "Learning-based animation of clothing for virtual try-on," Computer Graphics Forum: Journal of the European Association for Computer Graphics, vol. 17, no. 4, pp. 409-420, 2019.

[8] S. Hartanto and I. E. Y. Wiryanto, "Digitalisasi ppknjbpl," Journal PkM Pengabdian Kepada Masyarakat, vol. 3, no. 3, p. 301, 2020.

[9] A. Y. Alqahtani and A. A. Rajkhan, "Mixtures of normal distributions: application to diving suit fitting," Journal of Advanced Applied Scientific Research, vol. 2, no. 6, pp. 12-22, 2020.

[10] M. R. Minar, T. T. Tuan, and H. Ahn, "CloTH-VTON: Clothing Three-Dimensional Reconstruction for Hybrid Image-Based Virtual Try-ON," IEEE Access, vol. 9, 2021.

[11] Y. X. Wang and Z. D. Liu, "Virtual clothing display platform based on CLO3D and evaluation of fit," Journal of Fiber Bioengineering and Informatics, vol. 13, no. 1, pp. 37-49, 2020.

[12] D. Ofir and Y. Yanir, "Evaluating the thermal protection provided by a $2-3 \mathrm{~mm}$ wet suit during fin diving in shallow water with a temperature of $16-20^{\circ} \mathrm{C}$," Diving and Hyperbaric Medicine Journal, vol. 49, no. 4, pp. 266-275, 2019.

[13] S. Choi, W. Jo, Y. Jeon et al., "Multi-directionally wrinkle-able textile OLEDs for clothing-type displays," Npj Flexible Electronics, vol. 4, no. 1, pp. 516-523, 2020.

[14] H. Y. He and H. Liu, "Application practice of virtual try-on technology in garment design," China Textile Leader, vol. 4, no. 1, pp. 80-83, 2020.

[15] X. Li, "Research on fashion design system based on virtual reality technology," Video Engineering, vol. 42, no. 12, pp. 95-99, 2018. 